\title{
Title: Failed manual removal of the placenta after vaginal delivery
}

Johanna Bjurström ${ }^{1}$, Sally Collins ${ }^{2}$, Jens Langhoff-Roos ${ }^{1}$, Johannes J. Duvekot ${ }^{3}$, Karin Sundberg ${ }^{4}$, Annemette Jørgensen ${ }^{5}$, Lene Groenbeck ${ }^{1}$ for the EW-AIP*.

${ }^{1}$ Department of Obstetrics and Gynaecology, Copenhagen University Hospital, Rigshospitalet, Denmark ${ }^{2}$ Nuffield Department of Obstetrics \& Gynaecology, University of Oxford, The United Kingdom

${ }^{3}$ Department of Obstetrics and Gynaecology, Division of Obstetrics and Prenatal Medicine, Erasmus MC, University Medical Center Rotterdam, The Netherlands

4 Department of Fetal Medicine, Copenhagen University Hospital, Rigshospitalet, Denmark

${ }^{5}$ Department of Obstetrics and Gynecology, Aalborg University Hospital, Denmark

* The European Working group on Abnormally Invasive Placenta, EW-AIP, is a collaboration from 11 European university hospitals working in the field of abnormally invasive placentation. The EW-AIP was founded in 2008, their website is at http://www.ew-aip.org.

\section{Corresponding author:}

Johanna Bjurström

Obstetric Department

Blegdamsvej 9

2100 Copenhagen OE

johannabjurstrom@gmail.com

tlf no.: +4528205349

ORCID-ID 0000-0001-6496-267X

Word count main body: 4664 


\section{ABSTRACT}

Purpose: A retained placenta after vaginal delivery where manual removal of placenta fails is a clinical challenge. We present six cases that illustrate the heterogeneity of the condition and discuss the etiology and terminology as well as the clinical management.

Methods: Members of the European Working group on Abnormally Invasive Placenta (EW-AIP) were invited to report all recent cases of retained placenta that were not antenatally suspected to be abnormally adherent or invasive but could not be removed despite several attempts at manual removal. Results: The six cases from Denmark, the Netherlands and the UK provide examples of various treatment strategies such as ultrasound-guided vaginal removal, removal of the placenta through a hysterotomy and just leaving the placenta in situ. The placentas were all retained and clinically seemed to be abnormally adherent, but it was only possible to diagnose abnormal invasion in the one case which had a histopathological diagnosis of increta. Based on these cases we present a flow chart to aid clinical management for future cases.

Conclusion: We need properly defined stringent terminology for the different types of retained placenta, as well as improved tools to predict and diagnose both abnormally invasive and abnormally adherent placentas. Clinicians need to be aware of the options available to them when confronted by the rare case of a retained placenta in a patient who is not bleeding.

\section{Word count: 183}

\section{KEYWORDS}

- Maternal mortality and morbidity

- Obstetric complications of pregnancy

- Operative obstetrics

- $\quad$ Placenta 


\section{INTRODUCTION}

Retained placenta (RP) after vaginal delivery is a complication known to cause maternal morbidity and mortality. The immediate hazard of RP and subsequent attempts at manual removal is severe postpartum hemorrhage (PPH). Emergency hysterectomy, circulatory collapse and maternal death may be a consequence. Long-term complications of PPH are anemia, failure to lactate and postpartum depression among others (1). The pathophysiology of the condition is heterogeneous. RP may be categorized as 1) a normal placenta entrapped due to a contracted cervix or a structural uterine abnormality, 2) a normal placenta still adherent due to failed contraction of the retroplacental myometrium, or 3) an abnormally adherent (accreta) or invasive (increta or percreta) placenta also known as 'placenta accreta spectrum' (PAS) (2). Consensus regarding the treatment of RP has proved hard to achieve, perhaps due to difficulty in discriminating between the aforementioned causes. However regardless of the cause, manual removal of placenta (MROP) under regional or general anesthesia is recommended when active management, i.e. uterotonics and controlled umbilical cord traction, of the third stage of labor fails, the placenta remains in situ and there is no strong antenatal suspicion of abnormally adherent or invasive placenta.

When the retained placenta is abnormally adherent (accreta) or abnormally invasive (increta/percreta), manual removal is extremely difficult or even impossible. In cases of retained placenta due to failed contraction or entrapment, manual removal is usually a simple and blind procedure.

The present article presents six patients with RP after vaginal delivery where manual removal was not possible despite several attempts by experienced obstetricians. There had been no antenatal suspicion of PAS in any of the cases, therefore attempting MROP was appropriate clinical practice as was abandoning the attempts when it became apparent that the placenta could not be removed safely. The women were hemodynamically stable, without excessive bleeding. The supervising clinicians therefore opted for uterus-preserving management.

This article has two aims: Firstly, we present and discuss the considerations for maneuvers and interventions following failed MROP in the hemodynamically stable patient. These situations are rare and heterogeneous, therefore it is highly unlikely that evidence based recommendations will become available, making case reports important. Secondly these cases help demonstrate the inadequacy of our 
current definition of, and classifications for, the types of retained placenta. The gold standard for diagnosis of PAS is histopathological examination, according to the presence and degree of trophoblastic invasion. This is however, not possible if the placenta is left in situ, in these cases diagnosis must solely rely on imaging findings (sonographic or magnetic resonance imaging (MRI)) and clinical presentation. Only one of our six cases had tissue available for histopathological examination, this demonstrated evidence of abnormal placentation. None of the cases presented had typical imaging signs for PAS when examined both antenatally and postnatally using ultrasound/MRI. A suggestion for a clinical grading system to assess severity of the abnormally adherent and invasive placenta was recently published by Collins et al (3). Using this system, the cases presented would be classified as either grade 2 which is defined as "MROP required and parts of placenta thought to be abnormally adherent" or grade 3 defined as "MROP required and the whole placental bed thought to be abnormally adherent" (3). 


\section{MATERIALS AND METHODS}

Members of the EW-AIP were invited to report all their recent cases of vaginal delivery with a retained placenta following failed attempts at MROP where the women were hemodynamically stable thereby allowing consideration of how to proceed without emergency hysterectomy. We collected a total of six cases from Denmark, the Netherlands and the UK. 


\section{RESULTS}

\section{Case 1: Successful removal of placenta from a septate uterus under ultrasound guidance}

A 33-year-old Gravida 2, Para 0, with a history of missed miscarriage treated by dilatation and curettage (D\&C). Previous hysterosalpingography had shown a possible occlusion of the right salpinx, and the patient recalled being told that she had a "heart-shaped" uterus. In the index pregnancy, the patient contacted the delivery ward twice close to term with a constantly burning pain on the right side of the uterine fundus. An ultrasound scan of this area showed no abnormalities. After she delivered, the placenta remained in situ. Manual and instrumental removal of placenta was attempted unsuccessfully twice within 24 hours and an intrauterine balloon device was inserted to minimize blood loss. Her vaginal bleeding was not excessive. On an ultrasound scan, a bicornuate uterus with a septum was seen and the placenta appeared to be in the right corner. There were no sonographic signs of abnormally invasive placentation. A third attempt was made to remove the placenta vaginally on the forth day postpartum. Under ultrasound guidance, the placenta was removed manually and by inserting tissue forceps into the plane of cleavage, carefully separating the remaining placental tissue from the uterine wall in multiple small fragments. Histologic examination of the placental tissue removed showed no abnormalities. The patient was discharged four days after the procedure with no signs of infection and minimal vaginal bleeding. A follow-up ultrasound scan three months later showed an empty partial septate uterus (ESHRE Class U2). The upper right corner of the now empty uterine cavity had a blurred lining between the endometrium and the myometrium, this was suspected to have been the site of the placental implantation. 
A 30-year-old Gravida 2, Para 1, had one previous vaginal term delivery complicated by MROP with a PPH of approximately $3000 \mathrm{ml}$. After the second delivery the placenta was again retained. Two experienced physicians attempted MROP unsuccessfully 55 minutes after delivery of the neonate. An intrauterine balloon tamponade was inserted, and the patient was stabilized. The blood loss at this point was $3500 \mathrm{ml}$. The balloon was removed the following day, and bleeding was not excessive. An ultrasound scan showed a retained placenta with no signs of abnormally invasive placentation. The patient received several blood transfusions and broad-spectrum antibiotics. Five days later another attempt to remove the placenta was made after informing the patient of the risk of emergency hysterectomy in case of severe bleeding. Tissue forceps and curettage were used under ultrasound guidance and the placental tissue was successfully removed in multiple small fragments. The patient was discharged the next day but returned the same evening with fever. She received another 24 hours of intravenous antibiotics, followed by seven days of oral antibiotics for suspected endometritis. There were no further adverse events. When followed up three months postpartum, the patient had no gynecological complaints; she was breastfeeding and had regular menstrual periods using combined oral contraceptive pill. On an ultrasound scan an area of retained placental tissue was seen in the uterine fundus, therefore the patient was booked for a hysteroscopy. Placental tissue was resected from the posterior wall of the cavity. Histopathological examination of the placental tissue removed by ultrasound-guided forceps and curettage, and subsequently by hysteroscopy, was not reported as demonstrating evidence of PAS. 
Case 3: Retained placenta left in situ after several attempts of manual removal. Placenta fully absorbed nine months postpartum without adverse events

A 36-year-old Gravida 8, Para 1, with a history of previous surgery to remove a septum from a bicornuate uterus, six miscarriages all treated by D\&C and a vaginal delivery preterm at 27 weeks. In the index pregnancy, she was scanned antenatally by a fetal medicine doctor with considerable expertise in diagnosis of PAS, they felt that there were no ultrasound signs suggestive of abnormal placentation. Labor was induced due to PPROM at 31 week's gestation. After vaginal delivery the placenta did not separate spontaneously. Two experienced obstetricians attempted MROP unsuccessfully. The patient was given synthetic oxytocin and an intrauterine balloon device was inserted. Total blood loss was $900 \mathrm{ml}$. Bleeding was not excessive after removal of the balloon the following day. The placenta was left in situ and the patient was discharged having been given prophylactic oral antibiotics for seven days. The patient was scanned monthly until the placenta was completely reabsorbed. Once the placenta appeared fully absorbed on ultrasound, a serum human chorionic gonadotropin was measured which was was negative. This process took nine months and 11 days. Her blood loss during the follow-up period was minimal and there were no adverse events. 
Case 4: Placenta left in situ after attempted manual removal. Patient readmitted nine weeks postpartum with sepsis unresponsive to antibiotics. The placenta was successfully removed with tissue forceps under ultrasound guidance.

A 28-year-old Gravida 2, Para 0, with a history of previous first trimester termination of pregnancy by D\&C. After a spontaneous vaginal delivery at term, the placenta did not detach and two experienced obstetricians attempted MROP unsuccessfully. During the procedure, the patient bled approximately 400 $\mathrm{ml}$. The patient received prophylactic intravenous antibiotics during the procedure and for the first 24 hours. An ultrasound was performed three days postpartum which showed no signs of abnormally invasive placentation. The patient was discharged without antibiotics with the intention of monthly follow-up until the placenta was fully absorbed. However, nine weeks later, she was admitted with signs of sepsis (mildly raised white blood cell count, intermittently increased (spiking) temperatures and intermittent tachycardia to $110 \mathrm{bpm}$ ). She had no vaginal bleeding or lower abdominal pain. An ultrasound scan showed that the placental bed was now poorly perfused. She remained stable and as after three days of broad-spectrum intravenous antibiotics the symptoms had not resolved, MROP was attempted again. The placental tissue was successfully removed under ultrasound guidance with sponge forceps and gentle manual exploration of the cavity. Ultrasound examination at the end of the procedure demonstrated a clear endometrial echo with no evidence of any retained tissue. The patient made a good recovery and was discharged three days later with no vaginal bleeding. A subsequent serum human chorionic gonadotropin level was negative. Histology demonstrated necrotic tissue of placental origin with no other findings of note. 


\section{Case 5: Retained placenta in a bicornuate uterus removed by hysterotomy}

A 33-year-old, Gravida 3, Para 1 with a history of a missed miscarriage treated by an uncomplicated D\&C and an uncomplicated vaginal delivery at 36 week's gestation with $300 \mathrm{ml}$ of blood loss. After another vaginal delivery at 37 week's gestation the umbilical cord ruptured during controlled cord traction. Forty minutes later, the patient was transferred to the operating theatre. Two experienced consultants unsuccessfully attempted MROP. Blood loss had by then accumulated to $2000 \mathrm{ml}$. An intrauterine balloon device was inserted and sulprostone, a prostaglandin analogue, was administered, which stopped the bleeding. As the patient was haemodynamically stable she was transferred to a tertiary care hospital where an MRI scan was performed of the lower abdomen shortly after arrival. This showed the placenta located in the fundal part of the uterus with no signs of abnormally invasive placentation. More than ten hours after delivery, another attempt of MROP was performed. Bleeding was minimal after deflating the balloon tamponade, but the placenta could not be reached vaginally as the cervical dilatation was only three centimeters. A laparotomy was performed through a Pfannenstiel incision. The uterus had a bicornuate shape (ESHRE Class U2) with an enlarged left side where the placenta was located. A median incision was made in the uterus and the placenta was removed in multiple fragments from the left uterine horn. The uterine wall was sutured in layers. The estimated blood loss from the procedure was $4000 \mathrm{ml}$. The placental weight was 323 grams. No signs of abnormally invasive placentation were found when the placental tissue was sent for histological examination. 
Case 6: Sepsis after multiple attempts at manual removal, retained placental tissue finally removed by laparotomy demonstrating adherence to a leiomyoma.

A 34-year-old Gravida 2, Para 1, had one previous uncomplicated vaginal delivery at term. At 33 weeks into the index pregnancy she experienced preterm prelabor rupture of membranes (PPROM) followed by a spontaneous vaginal delivery 3 days later. The placenta was retained. Two experienced obstetricians performed an unsuccessful attempt of MROP. The following day an ultrasound showed the entire placenta in the right upper corner of the uterine fundus. Abnormally invasive placentation was suspected due to a thin uterine wall. Therefore, the patient was transferred to another tertiary care center with greater expertise in managing PAS. Manual and instrumental removal of the placenta was attempted twice unsuccessfully. An ultrasound scan as well as a magnetic resonance imaging (MRI) scan confirmed large amounts of retained placental tissue in the right corner of the uterine fundus. As she was stable and not bleeding excessively the decision was made to manage her conservatively and leave the placenta in situ. The patient was then referred back to her original delivery unit where she received prophylactic broadspectrum antibiotics and several blood transfusions. She was discharged home but returned 26 days postpartum with a fever and vaginal discharge. She had no vaginal bleeding or lower abdominal pain. Intravenous broad-spectrum antibiotics were administered and a CT scan confirmed retained placental tissue. A hysteroscopy was performed. The right tubal ostium was completely blocked by necrotic placental tissue. Resection of the tissue was not possible; therefore the surgery was converted to a laparotomy. The right uterine corner, which was grossly enlarged to approximately $8-10 \mathrm{~cm}$ in diameter, and a normal right salpinx were removed. The remaining uterus was sutured in layers. Postoperatively the patient received antibiotics and blood transfusions and was discharged few days after surgery. Histological examination of the resected myometrium with attached placenta concluded that the placenta had been located in the upper right side of the uterine cavity, infiltrating the myometrium, this was therefore classified as placenta increta. Furthermore, near the right ostium there was a $5 \mathrm{~cm}$ large intramural leiomyoma to which the placenta was adherent in an accrete manner. The right salpinx, fimbria and uterine corner were normal. 


\section{DISCUSSION}

When placenta is retained after vaginal delivery, there may be several underlying pathophysiological causes. It might be entrapped due to a closing cervix or uterine anomaly, it might not have separated due to failed retroplacental myometrial contraction or it might be an abnormally adherent or invasive placenta. This case series presents patients with the following common characteristics; 1) no antenatal suspicion of an abnormally invasive placenta, 2) a retained placenta after vaginal delivery, 3) failed attempt(s) at MROP by experienced obstetricians and 4) no excessive bleeding, allowing various uteruspreserving management strategies to be considered. These cases are rare and management differed based on expertise, attitude and available resources. None of the cases have been previously published, and to the authors' knowledge, only two similar case-reports have been published (4-6). Based on our cases we designed a flow chart to aid consideration of management after failed manual removal of the placenta (Figure 1.)

\section{Management of the cases}

When MROP following a vaginal delivery fails in a hemodynamically stable patient without ongoing bleeding, an intrauterine balloon tamponade should be considered to buy time to either decide on a management strategy or transfer the patient to a tertiary care facility. Advantages of referral to a tertiary facility include having access to advanced treatment modalities, such as pelvic arterial embolization and availability of specialists experienced in complex pelvic surgery (usually gynecological oncologists). Seeking advice from an expert in diagnostic imaging of PAS is also crucial for deciding further management including assessing the appropriateness of further attempts to remove the placenta. Irrespective of the chosen modality, imaging can determine whether the placenta is showing signs of abnormal invasion and can map out the cavity of an anomalous uterus. If subsequent imaging shows signs of PAS, a decision on management should be made after consideration of the expertise and resources available, with full consultation with the woman herself to assess her desire for future fertility. Options include; laparotomy with placental removal through a hysterotomy +/- local resection of the endometrium, a hysterectomy with the placenta left in situ, or conservative management with the 
placenta left to reabsorb. If imaging shows no signs of PAS, further ultrasound guided vaginal attempts at removal of the placenta should be considered.

If the placenta is removed through a hysterotomy, the patient will have an increased risk of abnormally invasive placentation in future pregnancies (7). If MROP fails, bleeding is minimal and there are no signs of infection conservative management by leaving the placenta in situ to be reabsorbed may be an option. This strategy was used in two cases; in one the strategy proved successful but it took nine months before the placenta had resolved, in the other, the patient developed sepsis. Factors to be taken into consideration before leaving the placenta in situ are the risk of complications such as infection or hemorrhage, potential compliance if complications arise, logistics such as distance from the patient's home to a hospital and the woman's desire for future fertility.

\section{Complications}

The two main complications in these cases were hemorrhage and infection. Blood loss is an important parameter when comparing treatment strategies however too few cases are presented here to be able to draw definitive conclusions regarding which management strategy best minimizes blood loss. The patient with the greatest blood loss was case number five; treated by removing the placenta from a bicornuate uterus through a hysterotomy the same day as the delivery. She bled approximately $2000 \mathrm{ml}$ while attempting MROP and an additional $4000 \mathrm{ml}$ during the laparotomy. The patient with the smallest blood loss was case number four; initially treated conservatively, however readmitted nine weeks postpartum due to sepsis requiring the placenta to be removed vaginally. She bled minimally prior to discharge, and approximately $400 \mathrm{ml}$ during the vaginal removal.

Four of the six cases developed fever and intrauterine infection at some point in the course of their treatment despite prophylactic antibiotics. This is a greater proportion than has been reported for planned conservative management due to antenatally diagnosed abnormally invasive placentation (8). Although these infections may be attributable to multiple attempts at removing the placenta vaginally, there is no clear consensus as to whether attempted MROP increases the risk of endometritis, and no studies have been conducted to determine the efficacy of routinely administered prophylactic antibiotics (9). 


\section{Risk factors for a retained placenta}

In these six cases, the complication of an abnormally adherent placenta was not anticipated including the one patient who had been screened for PAS. On further examination of the patient histories all the women had at least one risk factor for RP. One patient had previously had a retained placenta, which increases the risk of recurrence $(10,11)$. Three out of the six cases were preterm deliveries, a factor strongly associated with retained placenta (10-14). Previous uterine surgery is a known risk factor and although none had had a previous caesarean section or myomectomy, one had had a resection of a septum in a bicornuate uterus $(15,16)$. Uterine curettage, previous surgical termination and miscarriages have been identified as a risk factor for RP $(10-12,15,17)$. Four of the six patients had a history of at least one D\&C. Parity may be a risk factor however, studies report conflicting findings (10-15). Four of the women had had one previous baby with two being nulliparaous. Congenital uterine anomalies (CUAs) were present in at least three of the presented cases. The prevalence of CUAs in an unselected population is $5.5 \%$, and higher in a population with infertility and previous miscarriage (18). CUAs have been known to be associated with adverse outcomes in pregnancy, such as recurrent pregnancy loss, low birth weight, preterm birth, hypertensive disorders of pregnancy, malpresentation and cesarean delivery (19-22). To our knowledge, only one study has found an association between CUAs and RP, a finding our cases may support (21).

Use of intrauterine balloon, pelvic arterial embolization and methotrexate

An intrauterine balloon was used in four out of the six cases in an attempt to minimize blood loss. Although a recent review raises concerns about the lack of randomized controlled trials to prove the beneficial effect of intrauterine balloon (23), it has been implemented as an alternative in the surgical treatment for PPH in many obstetric settings due to a number of studies correlating its use to minimizing blood loss and avoiding peripartum hysterectomy (24-28). Current practice is to use an intrauterine balloon in an empty uterus, this is not how it was used in the cases presented in this article. In cases of PAS, where the placenta remains in situ, intrauterine balloon is generally not advised as it may pose a theoretical risk of tissue disruption and massive bleeding due to compression applied over damaged myometrium (29). This case series cannot comment on the efficacy or the safety of intrauterine balloon in these rare situations, however there were no adverse events. 
Pelvic arterial embolization (PAE) is a second-line treatment modality for PPH and is usually available in tertiary referral centers. Although a recent case series and a systematic review concluded that PAE is a highly efficient, safe, fertility preserving treatment, (30) this study does not include a single patient where the cause of PPH is retained placenta. The majority of the patients included, suffer from severe PPH due to uterine atony, the remainder bled secondary to genital hematomas, vaginal tears or arterio-venous malformations. When conducting a search using the MeSH terms "pelvic arterial embolization" and "retained placenta" found no relevant publications. We do not believe that pelvic arterial embolization would have been an appropriate treatment modality for any of the presented cases as they did not have ongoing bleeding.

Methotrexate (MTX) is a pharmacological treatment modality that has been applied as an adjunct to attempting conservative management of PAS, while awaiting reabsorption or expulsion (31-35). MTX inhibits the folic acid pathway; thereby targeting rapidly dividing cells, such as trophoblasts. It is therefore more commonly used for ectopic pregnancies and gestational trophoblastic diseases. That MTX can quicken reabsorption or expulsion of a postpartum placenta that is no longer growing seems biologically implausible. Senthiles et al. who have published the largest cohort of conservatively treated patients to date with PAS conclude that there is no convincing evidence of the drug's efficacy in these difficult cases (8). MTX is however contraindicated in breastfeeding and more importantly has potentially serious adverse effects, such as pancytopenia or nephrotoxicity. Intraumbilical MTX administration and finally multi-organ failure was the cause of the only maternal death in the aforementioned cohort of 167 patients. We do not believe that MTX should have been used in any of the presented cases.

\section{Limitations}

The major limitation of this case series is its size with only six cases reported. Due to the way the cases were collected no comment can be made regarding the incidence of this situation. The EW-AIP however, is made up of over 40 clinicians from 11 European countries all working in tertiary referral units specializing in PAS. The fact that when all of them were asked to report any cases only six were found means it is likely to be a relatively rare situation. Based on the heterogeneity of the cases, it is not possible 
to comment on the superiority of one treatment over the other, therefore we sought to report the different strategies which had been employed. As a result of this process we have developed the flow chart (Fig 1) to aid clinicians when faced the same situation. In terms of future fertility, to date there has been no follow-up of the patients' and therefore we cannot comment on any subsequent pregnancies.

\section{Challenges in terminology and classifications of the retained placenta}

The second aim of this article is to illustrate the inadequacy of the current definitions of and terminology used for the placenta that will not detach in the third stage of labor. The terms 'morbidly adherent placenta' (MAP) $(29,36)$, 'abnormally invasive placenta' (AIP) (3), 'placenta accreta spectrum' (PAS) (7) or just 'placenta accreta' are used as collective terms for the histopathological diagnoses of placenta accreta, increta and percreta. The degree of abnormal trophoblastic attachment or invasion defines the exact diagnosis; accreta is abnormally adherent, with the villi attaching directly to the myometrium without intermediate decidua, increta and percreta are abnormally invasive, with the villi invading into the myometrium in increta, and through the serosal layer in percreta (7).

These diagnoses should be made prenatally as this has been clearly shown to decrease maternal morbidity and mortality (37). This requires an initial diagnosis based on imaging findings (sonography and/or MRI). The sensitivity and specificity of sonography for detecting PAS are generally good, however the parameters differ depending on the expertise of the operator and the selection of the patient population (7). A vast number of studies on the sonographic appearance of PAS have been published, however there is no consensus on which sonographic signs should be used and how they can be described in an objective, reproducible manner with an acceptable inter-observer variability (38). High sensitivity is crucial as antenatal detection of PAS has a significant impact on the outcome for the patient. However specificity is also important as a false positive diagnosis may come with the price of a vertical laparotomy followed by a hysterectomy costing the patient her fertility (3). MRI is often used to confirm the sonographic diagnosis and to map the topography of the placental tissue in order to aid in planning the surgical intervention. However, whether MRI can improve diagnostic accuracy or outcome has not been proven to date (29). When PAS is detected prenatally the general recommendation is a planned, preterm caesarean section, leaving the placenta in situ prior to either performing a hysterectomy or attempting conservative management, i.e. closing the uterotomy after delivering the neonate and then 
leaving the placenta to reabsorb. Attempts to forcibly remove the placenta are strongly advised against as it may cause massive hemorrhage (36).

If there is no material available for histopathological diagnosis and there has been no imaging performed prenatally specifically to detect PAS, there only remains clinical diagnosis, which is highly subjective and variable. Authors of a recent review of PAS reported that "most authorities only consider cases that require additional surgical interventions to control bleeding, such as hysterectomy, uterine curettage, and embolization of pelvic vessels, to have clinical evidence of accreta" (7) whilst another paper recently published on behalf of NFOSS only considered cases requiring a blood transfusion and laparotomy to be accreta (Thurn et al 2016 BJOG, 123:1348-55). As its name suggests, PAS is a spectrum disorder ranging from abnormal adherence to the full blown invasion of percreta with the least severe, adherent end of the spectrum currently proving to be the hardest to define. In response to this issue an attempt has recently been made to generate a clinically relevant grading system for assessment of severity of PAS (3). Using this scale, all of of the cases would score at least grade 2 (abnormal adherence in parts of the placental bed on attempted MROP) if not grade 3 (abnormal adherence of the entire placental bed on attempted MROP) depending on the exact findings of the obstetrician at the time of MROP. Data is available on this for cases 3 and 4 confirming that they were considered to be grade 3 by the obstetrician attempting the MROP. Although this confirmation is not available for the other four cases it is most likely that they were grade 3 as if they were only partially adherent they would have probably partially separated during the attempt leading to significant bleeding. Therefore, using this scale, all of the cases would be classed as PAS most likely grade 3 .

So how should we define our cases? Should they be regarded as part of the PAS as the clinical grading scale suggests? Four of the patients had their placentas removed in multiple fragments, which suggests abnormal adherence, and one had histopathological findings of placenta accreta and increta, therefore this might seem appropriate. In the absence of histopathological results should the clinical presentation of a placenta which cannot be removed manually suffice to make the diagnosis of PAS? We believe that if the accoucher is experienced and able to insert their hand into the uterus far enough to grasp the placenta yet still cannot remove it, entrapment of a normally separated placenta can be ruled out in a normally formed uterus. Therefore, the most likely reason for an experienced obstetrician abandoning a MROP with the placenta still in situ is failure to find an adequate plane of cleavage to facilitate separating the placenta 
from the Uterus. This is highly unusual and suggests abnormal adherence to the uterine wall fitting with at least a grade three on the suggested clinical grading scale.

One thing is clear if high quality research is to continue into the spectrum of placenta accreta, just diagnosing it in a binary manner (PAS or not PAS) is insufficient. Agreement is urgently needed on a way to distinguish between (a) a normal placenta, (b) an abnormally adherent placenta and (c) an invasive placenta that is clinically relevant and can be employed in the absence of histological results. The only such classification that the authors are aware of is the previously published clinical grading scale for PAS (3) which has been adopted by the EW-AIP and is being used to grade severity in the data that they collect.

\section{CONCLUSIONS}

When a retained placenta cannot be removed manually, the patient is hemodynamically stable and the bleeding is under control we recommend ultrasonography or MRI to detect abnormally invasive placentation. Provided there are no signs of abnormal placentation an additional manual or instrumental attempt to remove the placenta vaginally can be considered using perioperative ultrasound guidance. If the second attempt fails, the options are to perform a laparotomy with either resection or hysterectomy, or to leave the placenta in situ, taking into account the long term follow up including risk of sepsis or hemorrhage after discharge of the patient. These strategies were associated with successful outcomes in this case series. No international consensus is available for the clinical diagnosis of PAS in the absence of histopathological data, however, using the clinical grading scale employed by the EW-AIP, all the cases presented could be regarded as PAS grade 2 or 3 . A flow chart has been devised by the authors to help facilitate management of similar cases in the future. 


\section{FIGURE CAPTION}

Fig. 1 A flow chart to aid management strategy for these rare but complicated patients based on the six cases presented in the article. MROP: Manual removal of placenta, MRI: Magnetic resonance imaging, AIP: Abnormally invasive placentation 


\section{CONTRIBUTION OF AUTHORSHIP:}

The authors all take responsibility for the paper as published.

J Bjurström: Protocol/project development, Data collection, Manuscript writing

J J Duvekot: $\quad$ Protocol/project development, Data collection, Manuscript editing

K Sundberg: $\quad$ Data collection, Manuscript editing

A Jørgensen: $\quad$ Data collection, Manuscript editing

S Collins: $\quad$ Data collection, Manuscript editing

J Langhoff-Roos: Protocol/project development, Data collection, Manuscript editing

L Groenbeck: Protocol/project development, Data collection, Manuscript editing 
COMPLIANCE WITH ETHICAL STANDARDS

FUNDING: This study received no funding

CONFLICT OF INTERESTS: The authors declare that they have no conflicts of interests to disclose.

ETHICAL APPROVAL: Informed consent to publish patient histories in an anonymous form was obtained from all six patients included. Therefore, approval from national ethics committees is not needed. 


\section{REFERENCES}

1. Evensen A, Anderson JM, Fontaine P. Postpartum Hemorrhage: Prevention and Treatment. Am Fam Physician. 2017;95(7):442-9.

2. Herman A. Complicated third stage of labor: time to switch on the scanner. Ultrasound Obstet Gynecol. 2000;15(2):89-95.

$3 . \quad$ Collins SL, Stevenson GN, Al-Khan A, Illsley NP, Impey L, Pappas L, et al. Three-Dimensional Power Doppler Ultrasonography for Diagnosing Abnormally Invasive Placenta and Quantifying the Risk. Obstet Gynecol. 2015;126(3):645-53. 4. Bennett MJ, Townsend L. Conservative management of clinically diagnosed placenta accreta following vaginal delivery. Aust N Z J Obstet Gynaecol. 2009;49(6):6479.

5. Lee D, Johnson J. Hysterotomy for retained placenta in a septate uterus: a case report. Case Rep Obstet Gynecol. 2012;2012:594140.

6. Shekhar S, Verma S, Motey R, Kaushal R. Hysterotomy for retained placenta with imminent uterine rupture in a preterm angular pregnancy. Acta Obstet Gynecol Scand. 2010;89(12):1615-6.

7. Silver RM, Barbour KD. Placenta accreta spectrum: accreta, increta, and percreta. Obstet Gynecol Clin North Am. 2015;42(2):381-402.

8. Sentilhes L, Ambroselli C, Kayem G, Provansal M, Fernandez H, Perrotin F, et al. Maternal outcome after conservative treatment of placenta accreta. Obstet Gynecol. 2010;115(3):526-34.

9. Chongsomchai C, Lumbiganon P, Laopaiboon M. Prophylactic antibiotics for manual removal of retained placenta in vaginal birth. Cochrane Database Syst Rev. 2014(10):CD004904.

10. Endler M, Grunewald C, Saltvedt S. Epidemiology of retained placenta: oxytocin as an independent risk factor. Obstet Gynecol. 2012;119(4):801-9.

11. Owolabi AT, Dare FO, Fasubaa OB, Ogunlola IO, Kuti O, Bisiriyu LA. Risk factors for retained placenta in southwestern Nigeria. Singapore Med J. 2008;49(7):5327.

12. Adelusi B, Soltan MH, Chowdhury N, Kangave D. Risk of retained placenta: multivariate approach. Acta Obstet Gynecol Scand. 1997;76(5):414-8.

13. Combs CA, Laros RK, Jr. Prolonged third stage of labor: morbidity and risk factors. Obstet Gynecol. 1991;77(6):863-7.

14. Coviello EM, Grantz KL, Huang CC, Kelly TE, Landy HJ. Risk factors for retained placenta. Am J Obstet Gynecol. 2015;213(6):864 e1- e11.

15. Ashwal E, Melamed N, Hiersch L, Wiznitzer A, Yogev Y, Peled Y. The incidence and risk factors for retained placenta after vaginal delivery - a single center experience. J Matern Fetal Neonatal Med. 2014;27(18):1897-900.

16. Belachew J, Cnattingius S, Mulic-Lutvica A, Eurenius K, Axelsson O, Wikstrom AK. Risk of retained placenta in women previously delivered by caesarean section: a population-based cohort study. BJOG. 2014;121(2):224-9.

17. Panpaprai P, Boriboonhirunsarn D. Risk factors of retained placenta in Siriraj Hospital. J Med Assoc Thai. 2007;90(7):1293-7.

18. Chan YY, Jayaprakasan K, Zamora J, Thornton JG, Raine-Fenning N, Coomarasamy A. The prevalence of congenital uterine anomalies in unselected and highrisk populations: a systematic review. Hum Reprod Update. 2011;17(6):761-71. 
19.

Chan YY, Jayaprakasan K, Tan A, Thornton JG, Coomarasamy A, Raine-

Fenning NJ. Reproductive outcomes in women with congenital uterine anomalies: a systematic review. Ultrasound Obstet Gynecol. 2011;38(4):371-82.

20. Fox NS, Roman AS, Stern EM, Gerber RS, Saltzman DH, Rebarber A. Type of congenital uterine anomaly and adverse pregnancy outcomes. J Matern Fetal Neonatal Med. 2014;27(9):949-53.

21. Hiersch L, Yeoshoua E, Miremberg H, Krissi H, Aviram A, Yogev Y, et al. The association between Mullerian anomalies and short-term pregnancy outcome. J Matern Fetal Neonatal Med. 2016;29(16):2573-8.

22. Vaz SA, Dotters-Katz SK, Kuller JA. Diagnosis and Management of Congenital Uterine Anomalies in Pregnancy. Obstet Gynecol Surv. 2017;72(3):194-201.

23. Wright CE, Chauhan SP, Abuhamad AZ. Bakri balloon in the management of postpartum hemorrhage: a review. Am J Perinatol. 2014;31(11):957-64.

24. Doumouchtsis SK, Papageorghiou AT, Arulkumaran S. Systematic review of conservative management of postpartum hemorrhage: what to do when medical treatment fails. Obstet Gynecol Surv. 2007;62(8):540-7.

25. Georgiou C. Balloon tamponade in the management of postpartum haemorrhage: a review. BJOG. 2009;116(6):748-57.

26. Kayem G, Kurinczuk JJ, Alfirevic Z, Spark P, Brocklehurst P, Knight M. Specific second-line therapies for postpartum haemorrhage: a national cohort study. BJOG. 2011;118(7):856-64.

27. Laas E, Bui C, Popowski T, Mbaku OM, Rozenberg P. Trends in the rate of invasive procedures after the addition of the intrauterine tamponade test to a protocol for management of severe postpartum hemorrhage. Am J Obstet Gynecol.

2012;207(4):281 e1-7.

28. Revert M, Cottenet J, Raynal P, Cibot E, Quantin C, Rozenberg P. Intrauterine balloon tamponade for management of severe postpartum haemorrhage in a perinatal network: a prospective cohort study. BJOG. 2016.

$29 . \quad$ D'Antonio F, Palacios-Jaraquemada J, Lim PS, Forlani F, Lanzone A, TimorTritsch I, et al. Counseling in fetal medicine: evidence-based answers to clinical questions on morbidly adherent placenta. Ultrasound Obstet Gynecol. 2016;47(3):290301.

30. Ruiz Labarta FJ, Pintado Recarte MP, Alvarez Luque A, Joigneau Prieto L, Perez Martin L, Gonzalez Leyte M, et al. Outcomes of pelvic arterial embolization in the management of postpartum haemorrhage: a case series study and systematic review. Eur J Obstet Gynecol Reprod Biol. 2016;206:12-21.

31. Cirpan T, Sanhal CY, Yucebilgin S, Ozsener S. Conservative management of placenta previa percreta by leaving placental tissue in situ with arterial ligation and adjuvant methotrexate therapy. J Turk Ger Gynecol Assoc. 2011;12(2):127-9.

32. Heiskanen N, Kroger J, Kainulainen S, Heinonen S. Placenta percreta: methotrexate treatment and MRI findings. Am J Perinatol. 2008;25(2):91-2.

33. Lalchandani S, Geary M, O'Herlihy C, Sheil O. Conservative management of placenta accreta and unruptured interstitial cornual pregnancy using methotrexate. Eur J Obstet Gynecol Reprod Biol. 2003;107(1):96-7.

34. Mussalli GM, Shah J, Berck DJ, Elimian A, Tejani N, Manning FA. Placenta accreta and methotrexate therapy: three case reports. J Perinatol. 2000;20(5):331-4. 35. Pinho S, Sarzedas S, Pedroso S, Santos A, Rebordao M, Avillez T, et al. Partial placenta increta and methotrexate therapy: three case reports. Clin Exp Obstet Gynecol. 2008;35(3):221-4. 
36. Lim BH, Palacios-Jaraquemada JM. The morbidly adherent placenta--a continuing diagnostic and management challenge. BJOG. 2015;122(12):1673.

37. Tikkanen M, Paavonen J, Loukovaara M, Stefanovic V. Antenatal diagnosis of placenta accreta leads to reduced blood loss. Acta Obstet Gynecol Scand. 2011;90(10):1140-6.

38. Bhide A, Sebire N, Abuhamad A, Acharya G, Silver R. Morbidly adherent placenta: the need for standardization. Ultrasound Obstet Gynecol. 2017;49(5):559-63. $39 . \quad$ Perez-Delboy A, Wright JD. Surgical management of placenta accreta: to leave or remove the placenta? BJOG. 2014;121(2):163-9; discussion 9-70.

40. Urner F, Zimmermann R, Krafft A. Manual removal of the placenta after vaginal delivery: an unsolved problem in obstetrics. J Pregnancy. 2014;2014:274651. 